\title{
DEFECT MEASUREMENT USING INFRARED THERMOGRAPHY FOR MEASURING INVISIBLE AREA
}

\author{
S. C. KIM ${ }^{\dagger}$, H. C. JUNG, K. S. KIM*1 \\ ${ }^{1}$ Department of Mechanical System Engineering, Chosun University, Gwangju, Korea \\ ${ }^{\dagger}$ Presenting Author: bluerune@nate.com \\ *Corresponding Author: gsckim@chosun.ac.kr
}

\begin{abstract}
Defect inspection system for industrial applications takes the important portion. Non-destructive inspection method has been significantly improved. Infrared thermography, as one of method for non- destructive inspection, can provide relatively precise data and quick inspection time. This study, it was performed to measure defect according to the measurement limit of the nonvisible areas such as the back surface of the pipe using reflection plate. Proper material can be selected based on space limitation and thermal characteristics of target material that detection rate of defect can be improved. Detection of defect in non-visible area using the candidate materials for reflection plate was conducted.
\end{abstract}

KEYWORDS: Reflection plate, Infrared thermography, Invisible area

\section{INTRODUCTION}

Infrared thermography presents real-time images of temperature variation by detecting radiation energy from object surface. It has been used for thermal characteristic evaluation, thermal imaging on power system, defect inspection for composite material, evaluation for building insulation, medical diagnosis using body temperature, etc.

All objects, which are above absolute temperature, emit IR radiation energy. IR thermography is used to detect and measure thermal energy in IR spectrum from object. These IR energies are originated from vibration and rotation of molecule. As temperature of object is high, more movement is followed, and higher amount of infrared energy is radiated. This energy is detected by infrared thermography camera. IR thermography camera utilizes device to detect IR spectrum from object, and converts the amount of IR to temperature. It can be displayed as IR or thermal image. Therefore, IR thermography can show temperature distribution on the surface of object, and it can give temperature distribution at each point. Among non-contact, nondestructive testing techniques that can detect several wall-thinning defects quickly and simply, Infrared Thermography (IRT) has been studied for application $[1,2]$.

\section{EQUIPMENT AND OBJECTS}

\subsection{REFLECTION PLATE}

The reflection plate is made of brass, carbon steel, copper, pure iron and aluminum. The reflection plate size is $300 \mathrm{~mm} * 300 \mathrm{~mm}$. The specimens were selected to have high heat absorbing capacity and high probability of detecting defects of test specimens.

\subsection{WALL-THINNED PIPE SPECIMEN}

Behind flat plate test specimens, nine defects of different sizes were made of iron, stainless steel and aluminum. The depth of the defect is equal to $50 \%$ of the thickness of the specimen, and the defect length and height are different from each other. 


\section{EXPERIMENT METHODS}

When the object including the defect does not generate self-heating, it induces the temperature deviation in the defect part and the dry part while the object is heated by externally applying a stimulus through thermal irradiation such as a halogen lamp. Since all test specimens do not generate heat by itself, lock-in technique were applied using two halogen lamps with a maximum capacity of $1 \mathrm{~kW}$ from the outside. For the experiment, the infrared camera kept the average temperature $\left(23 \pm 0.5^{\circ} \mathrm{C}\right)$ and the air conditioner was used to keep the humidity constant. In order to prevent the reflection of light, KRYLON Ultra Flat Black was applied to maintain the 0.97 emissivity.

\section{RESULTS AND DISCUSSION}

As a result of the experiment using the reflection plate, the defect was detected. Since the lock-in technique has a higher definition of defects than the infrared thermography, some defects are similar to actual defects and have a clear shape and size, and other defects are more clearly and clearly detected. In order to observe only the temperature change of the object and to overcome the limitation of the post-processing method, more accurate results can be obtained by using the lock-in method.

\section{ACKNOWLEDGMENT}

This work was supported by the Korea Institute of Energy Technology Evaluation and Planning(KETEP) and the Ministry of Trade, Industry \& Energy(MOTIE) of the Republic of Korea (No. 20164030201290).

This work was supported by the National Research Foundation of Korea(NRF) grant funded by the Korea government(MSIP) (No. 2012M2A2A9035705)

\section{REFERENCES}

[1] K. J. Lee, H. S. Jang, H. C. Jung and K. S. Kim, "Quantitative out-of-plane deformation measurement of pressure vessel with the defect using shearography," Journal of the Korean Society for Precision Engineering, Vol. 23, No. 10, pp. 36-42 (2006)

[2] X. P. V. Maldague, Trends in Optical Nondestructive Testing and Inspection, P. K. Rastogi, D. Ibaudi, Editors Elsevier Science, Switzerland (2000) 